\title{
Increased nitric oxide levels in cerebellum of cachectic rats with Walker 256 solid tumor
}

\author{
Fabio Leandro Fenner ${ }^{1}$, Flavia Alessandra Guarnier ${ }^{2}$, Sara Santos Bernardes ${ }^{1}$, Leandra Naira Zambelli Ramalho ${ }^{3}$, \\ Rubens Cecchini ${ }^{2}$, Alessandra Lourenço Cecchini ${ }^{1}$ \\ ${ }^{1}$ Laboratório de Patologia Molecular, Universidade Estadual de Londrina (UEL), Londrina, ${ }^{2}$ Laboratório de Fisiopatologia e Radicais \\ Livres, Universidade Estadual de Londrina (UEL), Londrina, ${ }^{3}$ Departamento de Patologia e Medicina Legal, Universidade \\ de São Paulo, Ribeirão Preto, Brasil
}

\begin{abstract}
In cancer cachexia, the role of nitric oxide (NO) in the central nervous system remains unclear. Cerebellar degeneration has been reported in cancer patients, but the participation of NO has not been studied. Thus, this study investigated the mechanism of oxidative cerebellar injury in a time-course cancer cachexia experimental model. The cachexia index is progressive and evident during the evolution of the tumor. Nitric oxide and lipid hydroperoxidation quantification was performed using a very sensitive and precise chemiluminescence method, which showed that both analyzed parameters were increased after tumor implantation. In the day 5 group, NO was significantly increased, and this experimental time was chosen to treat the rats with the NO inhibitors N-nitro-L-arginine methyl ester (L-NAME) and aminoguanidine (AG). When treated with NO inhibitors, a significant decrease in both NO and lipid hydroperoxide levels occurred in the cerebellum. 3-nitrotyrosine was also analyzed in cerebellar tissue by immunohistochemistry; it was increased at the three experimental time points studied, and decreased when treated with L-NAME and AG. Besides demonstrating that lipid hydroperoxidation in the cerebellum of rats with cachexia increases in a time-dependent manner, this study is the first to describe the participation of NO and its oxidized product 3-NT in the cerebellum of cachectic rats bearing the Walker 256 solid tumor.
\end{abstract}

Key words: nitric oxide, oxidative stress, 3-nitrotyrosine, Walker 256 tumor, cerebellum.

\section{Introduction}

Cachexia is characterized by marked metabolic alterations leading to massive weight loss, anorexia, asthenia and anemia [25]. Additionally, there is an inappropriate increase in energy expenditure, defects in protein, carbohydrate and lipid metabolism and muscle loss. The role of oxidative stress in the mechanism of muscle wasting was investigat- ed in rats implanted with Walker 256 solid tumor [10], which leads to cachectic syndrome very quickly. Brain weight loss is also observed [6] and cerebellar degeneration with loss of Purkinje cells occurs in the course of neoplastic diseases [16]. Brain and nervous tissue are prone to oxidative damage for several reasons. The central nervous system (CNS) has a high rate of oxygen consumption, a high amount of poly-

\section{Communicating author:}

Alessandra L. Cecchini, Departamento de Patologia Geral - Centro de Ciencias Biologicas, Universidade Estadual de Londrina, Laboratorio de Patologia Molecular CEP 86051-990 Londrina, Brazil, phone: (+55-43) 3371-4529, fax: (+55-43) 3371-4267, e-mail: alcecchini@uel.br 
unsaturated fatty acid and also many neurotransmitters which are autoxidizable molecules [11].

Nitric oxide (NO) is a highly reactive gas molecule that can either function as a beneficial physiological agent utilized for essential functions such as vasodilation, ischemia protection, and neurotransmission, or as a pathological agent that causes or exacerbates diseases, such as septic shock, cardiac hypertrophy and diseases of the central nervous system. Whether NO is helpful or harmful depends on a variety of factors, such as the cellular environment in which NO is released, and the dose released $[4,8]$. In the CNS, nitric oxide and its redox related forms have been reported to be both neuroprotective and neurodestructive [3]. However, several groups have been unable to demonstrate NO's direct toxicity or if it may contribute to oxidative stress and oxidative lesions of the CNS.

As a neuroprotector, $\mathrm{NO}$ is able to promptly react with free radicals in the medium, which allows it to act as a chain reaction terminator [7]. Cytotoxic NO may cause nitrosative and oxidative stress [2]. The death of cells or the ionic gradient collapse during energy depletion may cause a massive release of glutamate by neurons, causing a prolonged increase of intracellular $\mathrm{Ca}^{2+}$, and overproduction of NO through nNOS [11]. According to Halliwell and Gutteridge [11], this excitotoxicity may become a vicious circle where more glutamate is released with concomitant generation of $\mathrm{O}_{2}{ }^{--}$and NO. As a result, there is overproduction of peroxynitrite $\left(\mathrm{ONOO}^{\bullet-}\right)$, one of the most potent reactive species.

In this study we characterized the participation of nitrosative and oxidative stress in paraneoplastic cerebellar degeneration in a Walker 256 tumor progression experimental model in rats.

\section{Material and methods}

\section{Animals}

Adult male Wistar rats (250-350 g; $n=6 /$ group) were obtained from the Animal House of the Biological Sciences Center at the Universidade Estadual de Londrina. The animals were given water and commercial food (Nuvilab CR1, Nuvital Nutrients Ltda., Curitiba, Brazil) ad libitum. The food intake was measured daily. The experimental protocol was approved by the Institutional Animal Care and Utilization Committee of our institution (protocol 7794/2011). In all respects, the protocols conformed to the Guide for the Care and Use of Laboratory Animals.

\section{Reagents}

All the chemicals were obtained from Merck (Darmstadt, Germany) or Sigma-Aldrich (St. Louis, USA) laboratories.

\section{Tumor implantation}

Rats were divided into six groups designated as controls and tumor hosts at three different days of tumor progression $\left(5^{\text {th }}, 10^{\text {th }}\right.$ and $\left.14^{\text {th }}\right)$. The last time of sacrifice was determined based on the average time of survival, which was 15 days after tumor implantation. Tumor hosts at day 5 of tumor progression were treated with two different NOS inhibitors (aminoguanidine hydrochloride - AG [50 mg/kg] or N-nitroL-arginine methyl ester - L-NAME $[20 \mathrm{mg} / \mathrm{kg}$ daily, i.p.]. The control group received a $0.5 \mathrm{ml}$ injection of PBS, and the tumor-bearing rats received a Walker 256 cell suspension $\left(8.0 \times 10^{7}\right.$ cells in $0.5 \mathrm{ml}$ of PBS) injected subcutaneously on the right hind limb flank. The treated day 5 group received the same cell suspension as well as NOS inhibitors. Tumor cells were maintained in our laboratory as described by Guarnier et al. [10]. Three additional groups were included: a pair-fed group, where 6 animals inoculated with PBS were fed with the same amounts of food consumed by the tumor groups for 14 days, and a control group treated with NOS inhibitors for 5 days. On days 5,10 and 14 after subcutaneous tumor implantation, the animals in both tumor groups were weighed and killed by decapitation. The tumor was carefully excised and weighed. The cachexia index was determined considering initial and final body weight, tumor weight and body weight gain in control groups, according to the formula: [(initial body mass - final body mass + body mass gain of control) / (initial body mass + body mass gain of control)] $x$ 100\% [10]. The cachexia index was calculated in order to determine the pattern of general wasting. The cerebellum was rapidly excised, weighed, and stored in liquid nitrogen until use (at most, 60 days of storage). Since the pair-fed group did not develop cachexia, all experimental results were compared to the control.

\section{Tissue preparation}

Cerebellum was placed on ice and gently homogenized manually for approximately 2 minutes in a Potter glass tube in $\mathrm{Na}_{2} \mathrm{CO}_{3} 2 \mathrm{mM}, \mathrm{pH} 8.5$, previously bubbled with $\mathrm{N}_{2}$ to remove $\mathrm{O}_{2}$. The samples 
were centrifuged at $1,500 \times \mathrm{g}$ for $10 \mathrm{~min}$ at $4^{\circ} \mathrm{C}$ in a Jouan BR4i Multifunction centrifuge (Thermo Electron Corporation, USA). The supernatants were distributed in 4 Eppendorf tubes and kept in ice away from light until the moment of analysis. For the NO and lipid hydroperoxide quantification, samples were prepared in the concentration of 1.25 and $15 \mathrm{mg} / \mathrm{ml}$, respectively.

\section{Quantification of nitric oxide}

The production of nitric oxide (NO) was quantified in the cerebellum in the experimental animals through the technique based on the reaction of chemiluminescence initiated in the presence of $\mathrm{H}_{2} \mathrm{O}_{2}$ and luminol. The chemiluminescence was detected at $302 \mathrm{~nm}$ as described by Kikuchi et al. [14], modified by Terra et al. [22]. Briefly, a solution composed by $360 \mu \mathrm{M}$ Luminol/3 mM DFX was added to an equal volume of solution of $200 \mathrm{mM} \mathrm{H}_{2} \mathrm{O}_{2}$ and $1400 \mu \mathrm{l}$ of buffer $\left(2 \mathrm{mM} \mathrm{Na}_{2} \mathrm{CO}_{3}\right)$. Both solutions were prepared with the same buffer, $2 \mathrm{mM} \mathrm{Na}_{2} \mathrm{CO}_{3}, \mathrm{pH} 8.5$, previously bubbled with $\mathrm{N}_{2}$. The mixture was incubated at approximately $25^{\circ} \mathrm{C}$ under mild agitation for $5 \mathrm{~min}$. After this period, this solution $(180 \mu \mathrm{M}$ Luminol/1.5 mM DFX/200 $\mathrm{mM} \mathrm{H}_{2} \mathrm{O}_{2}$ ) was added to the sample $(1.25 \mathrm{mg} / \mathrm{ml})$ through a precision syringe (SGE, Australia). The chemiluminescence spectrum was monitored for 5 minutes using a luminometer model TD 20/20 (Turner Designs, USA) with detection capacity in wavelengths from 300 to $650 \mathrm{~nm}$ and $68.5 \%$ sensitivity. The luminometer was connected to a microcomputer through the program Spreadsheet Interface v1.0, making possible the registration of the emitted chemiluminescence. The program Origin v.7.5 was used for construction of the chemiluminescense curves. Samples were analyzed in quadruplicate.

\section{Cerebellum 3-nitrotyrosine immunohistochemistry}

The cerebellum preparations were also submitted to immunohistochemical analysis of 3-nitrotyrosine (3-NT), a protein nitrosative/oxidative stress

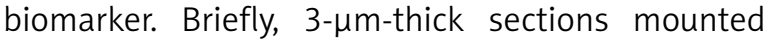
on poly-l-lysine-coated slides were deparaffinized, rehydrated, immersed in $10 \mathrm{mmol} / /$ citrate buffer, $\mathrm{pH}$ 6.0, and submitted to heat-induced epitope retrieval using a vapor lock for $45 \mathrm{~min}$. The slides were rinsed with phosphate-buffered saline (PBS) and immersed in 3\% hydrogen peroxide for $20 \mathrm{~min}$ to block endogenous peroxidase. Non-specific protein binding was blocked with normal serum (Vectastain Elite ABC Kit, Universal, Vector Laboratories Inc., Burlingame, CA, USA) for $30 \mathrm{~min}$. The sections were then incubated with monoclonal primary antibodies specific for nitrotyrosine (clone HM11; Santa Cruz Biotechnology, Santa Cruz, USA) for $2 \mathrm{~h}$ at room temperature $\left(25^{\circ} \mathrm{C}\right)$ in a humid chamber. Following washes in PBS, biotinylated pan-specific universal secondary antibody (Vectastain Elite ABC Kit, Universal, Vector Laboratories Inc.) was applied for $30 \mathrm{~min}$. Next, the slides were incubated with the avidinbiotin-peroxidase complex (Vectastain Elite ABC Kit, Universal, Vector Laboratories Inc.) for $30 \mathrm{~min}$ and developed with a NovaRed kit (Vector Laboratories Inc.) for $5 \mathrm{~min}$. The slides were counterstained with hematoxylin, dehydrated and mounted with Permount (Biomeda, Foster City, CA, USA). As negative controls, all specimens were incubated with an isotope-matched control antibody under identical conditions. The immunolabeling was considered to be positive when distinct red nuclear or cytoplasmic staining was present homogeneously. The percentage of nitrotyrosine-positive cells was obtained by Image J software developed at the U.S. National Institute of Health and available on the internet at http:// rsb.info.nih.gov/nih image. Results are presented as $\%$ mean \pm SEM.

\section{Determination of oxidative stress by highly sensitive chemiluminescence induced by tert-butyl hydroperoxide}

Reaction mixtures were placed in 2-ml luminescence tubes containing the following: cerebellum homogenate of control or experimental groups

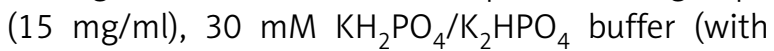
$120 \mathrm{mM} \mathrm{KCl}, \mathrm{pH} 7.4$ ), and $3 \mathrm{mM}$ tert-butyl hydroperoxide, in a final volume of $1 \mathrm{ml}$. The tert-butyl hydroperoxide-initiated chemiluminescence $(\mathrm{CL})$ reaction was assessed in a TD/20 20 luminometer (Turner Designs), with a response range of $300-650 \mathrm{~nm}$. The tubes were kept in the dark until the moment of assay, which was carried out in a room at $30^{\circ} \mathrm{C}$ $[9,17]$. For each animal, a 60 -min curve, where each point represented the differential smoothing of 600 readings, was obtained by interpolation. The results were expressed in relative light units/g tissue (RLU/g tissue). 


\section{Statistical analysis}

The results are shown as means \pm SEM. Two-way ANOVA with the Bonferroni post-hoc test was used to analyze the lipid peroxidation, $\mathrm{NO}$ and the entire chemiluminescence curve. Quantitative results were analyzed by one-way ANOVA with the Bonferroni post-hoc test. Statistical analysis was performed using GraphPad Prism 4.0 and 5.0 (GraphPad, San Diego, CA).

\section{Results}

\section{Characterization of cachexia}

Table I shows the cachexia index, mass of the tumor and the cerebellum absolute weight variation with or without AG and LNAME treatment on day 5. Animals with a cachexia index equal to or greater than $10 \%$ were considered as suffering from cachexia. The cachexia index is progressive and evident during evolution of the tumor. The animals did not present cachexia on D5, and when the animals were treated with aminoguanidine (D5AG) or L-NAME (D5LN) there was no difference when compared to D5. The same result could be seen in tumor weight, as the variation between D5, D5AG and D5LN was not significant. The cerebellum absolute weight did not change, although it showed an increase in weight variation in relation to the control group on day 5 and day 10 . When the day 5 group was treated with L-NAME (day 5LN) and aminoguanidine (day $5 A G)$ the percentage of weight variation was negative compared to the control, or the weight gain variation of the cerebellum was reverted by these treatments.

\section{Nitric oxide quantification by chemiluminescence and 3-nitrotyrosine by immunohistochemistry}

Figure 1 illustrates the chemiluminescence induced by $\mathrm{H}_{2} \mathrm{O}_{2}$-luminol for the quantification of $\mathrm{NO}$ in the cerebellum of control and tumor-bearing rats on day 5, day 10, day 14 (Fig. 1A) and day 5 treated with aminoguanidine (AG) and L-NAME (LN) (Fig. 1B). As observed in Figure 1A, the NO levels in cerebellum of the day 5 group was significantly increased compared to the control $(p<0.0001)$, so this experimental day was chosen to evaluate the effect of NO inhibitor treatment. The groups of animals pair-fed D5, D10 and D14 did not show levels of NO significantly higher compared to control PBS. Thus, it was demonstrated that the decrease in food intake does not interfere with increased levels of NO, and it was not subjected to further investigation. Figure $1 \mathrm{~B}$ shows the NO quantification in the cerebellum of 5-day tumor-bearing rats treated with inhibitors of cNOS (LNAME) and iNOS (aminoguanidine), D5AG and D5LN, respectively. The NO levels of D5AG and D5LN were significantly decreased compared to group D5 $(p<0.0001)$. The inhibition with LNAME is 1300 times greater than with AG. Figure 2 shows the increase of labeled area for 3-nitrotyrosine (3-NT) of the day $5(6.40 \pm 0.15 \%)$, day 10 $(10.99 \pm 0.098 \%)$ and day $14(14.12 \pm 0.14 \%)$ groups when compared to the control $(2.14 \pm 0.07 \%)$. When

Table I. Cachexia index, tumor and cerebellum weights in tumor-bearing rats in NO inhibitor treated and non-treated animals

\begin{tabular}{|lcccc|}
\hline & Tumor weight $(\mathrm{g})$ & Cachexia index $(\%)$ & \multicolumn{2}{c|}{ Cerebellum } \\
\cline { 3 - 5 } & & & Weight $(\mathrm{mg})$ & Percent variation \\
\hline Control & - & - & $0.287 \pm 0.007^{\mathrm{a}}$ & - \\
\hline D5 & $6.192 \pm 1.11^{\mathrm{a}}$ & $7.88 \pm 0.01^{\mathrm{a}}$ & $0.290 \pm 0.006^{\mathrm{a}}$ & +1.04 \\
\hline D10 & $22.057 \pm 2.55^{\mathrm{b}}$ & $14.02 \pm 1.05^{\mathrm{b}}$ & $0.306 \pm 0.01^{\mathrm{a}}$ & +6.60 \\
\hline D14 & $38.84 \pm 5.89^{\mathrm{c}}$ & $17.91 \pm 2.25^{\mathrm{b}}$ & $0.287 \pm 0.01^{\mathrm{a}}$ & 0.00 \\
\hline D5AG & $4.73 \pm 0.33^{\mathrm{a}}$ & $6.23 \pm 0.65^{\mathrm{a}}$ & $0.283 \pm 0.01^{\mathrm{a}}$ & -1.39 \\
\hline D5LN & $4.32 \pm 0.49^{\mathrm{a}}$ & $8.07 \pm 1.08^{\mathrm{a}}$ & $0.280 \pm 0.01^{\mathrm{a}}$ & -2.43 \\
\hline
\end{tabular}

Groups represent number of days after subcutaneous injection of $8.0 \times 10^{7}$ tumor cells with or without treatment with aminoguanidine (AG - 50 mg/kg daily, i.p.) or L-NAME (LN - $20 \mathrm{mg} / \mathrm{kg}$ daily, i.p.). Each group consisted of 6 animals. Control received an injection of $0.5 \mathrm{ml}$ of PBS. Cachexia index $=[($ initial body mass - final body mass + tumor weight + body mass gain of control)/(initial body mass + body mass gain of control)] $\times 100 \%$. Values are expressed as mean \pm SE. Positive values represent gain, and negative values decrease when compared with the control group. (-) in spaces means no comparison. In statistical evaluation, in the same column, different letters indicate statistical differences ( $p<0.05$ evaluated by one-way ANOVA, followed by Bonferroni's multiple comparison test), while the same letters indicate no statistical differences ( $n=6$ for all groups). 

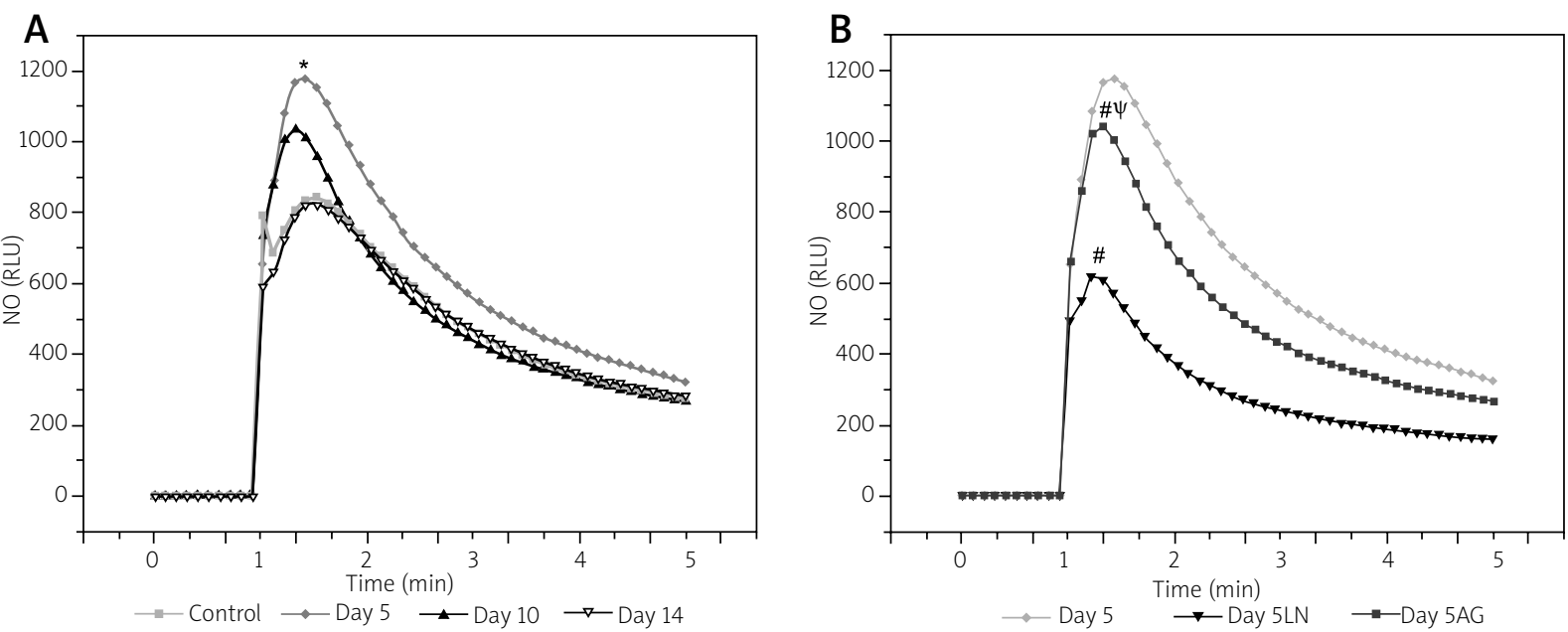

Fig. 1. Nitric oxide quantification by chemiluminescence. (A) Nitric oxide quantification of tumor-bearing rats on day 5, day 10, day 14. ${ }^{*} p<0.0001$ compared to control. (B) Effect of NOS inhibitors on the level of NO measured by chemiluminescence initiated by $\mathrm{H}_{2} \mathrm{O}_{2}$-luminol in cerebellum of tumor-bearing rats on day 5 , ${ }^{\#} p<0.0001$ compared to D5. ${ }^{\psi} p<0.0001$ compared to day $5 \operatorname{LN}$ ( $n=6$ for all groups).

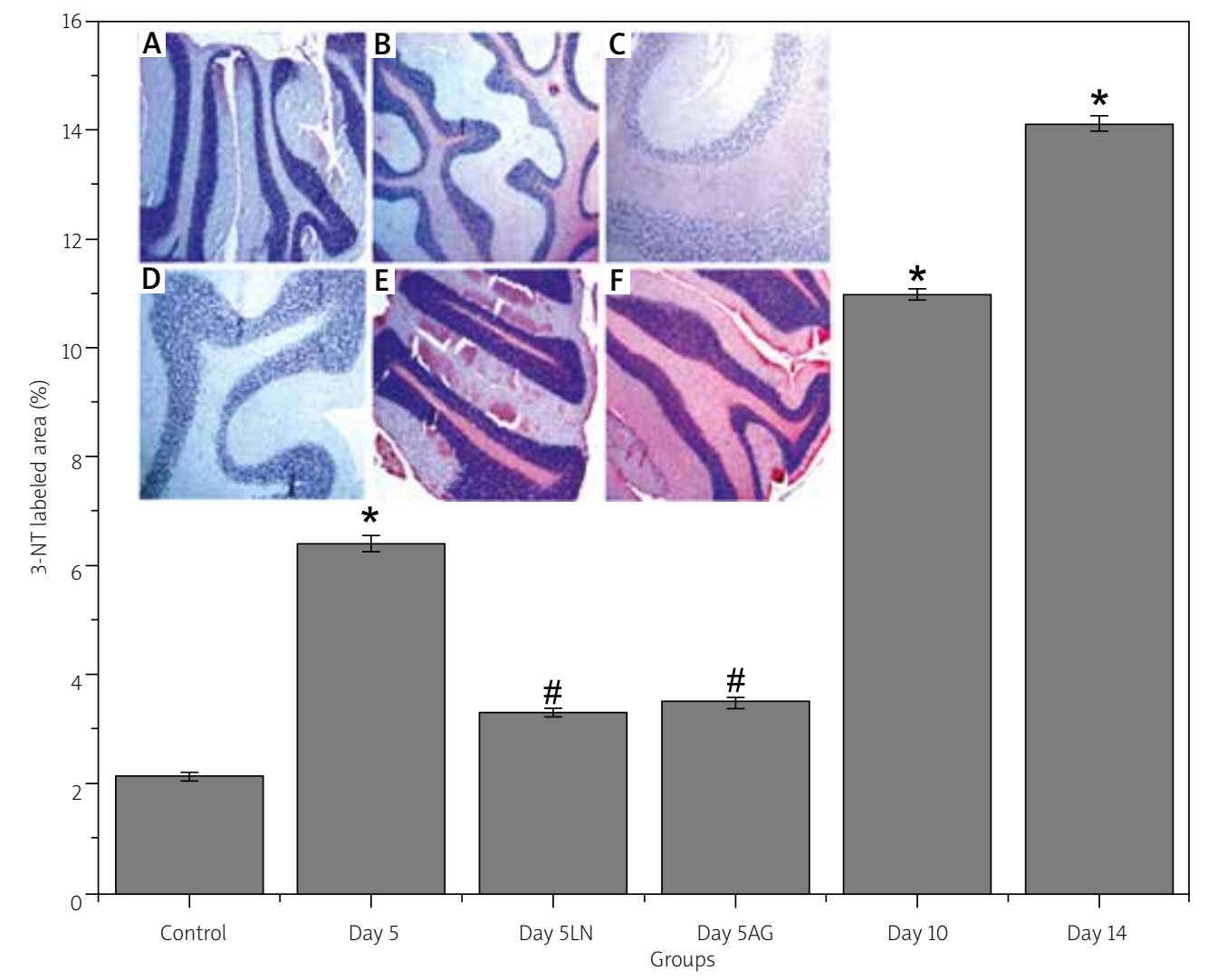

Fig. 2. 3-Nitrotyrosine (3-NT) immunohistochemistry in cerebellum. Groups were compared using one-way ANOVA with Bonferroni's post hoc test and $p<0.05$ was considered significant. ${ }^{*} p<0.05$ in relation to control group; ${ }^{*} p<0.05$ in relation to day 5 group. AG - aminoguanidine, L-NAME - N-nitro-L-arginine methyl ester. Above, representative photomicrographs (20x) of immunohistochemistry of 3-NT labeled area: A) control group; B) day 5; C) day 5LN; D) day 5AG; E) day 10; F) day 14 ( $n=6$ for all groups). 

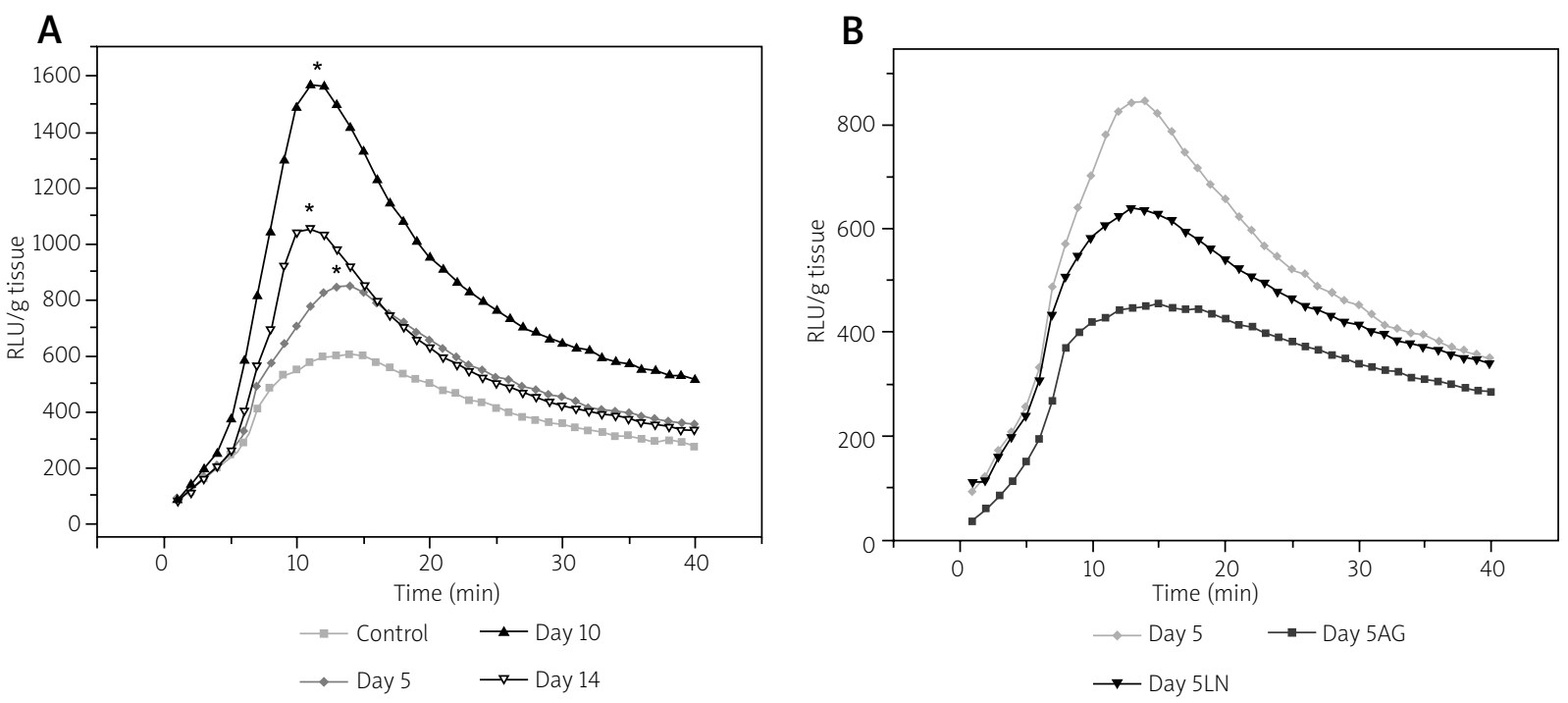

Fig. 3. Lipid hydroperoxide quantification of cerebellum determined by highly sensitive chemiluminescence induced by tert-butyl hydroperoxide. (A) Lipid hydroperoxide formation on day 5, day 10 and day 14 of cerebellum of tumor-bearing rats, ${ }^{*} p<0.0001$ compared to control. (B) Effect of NOS inhibitors on the levels of lipid hydroperoxides in the cerebellum of tumor-bearing rats on day $5 .{ }^{\#} p<0.0001$ compared to D5 ( $n=6$ for all groups).

D5 was treated with L-NAME $(3.61 \pm 0.11 \%)$ and AG (3.30 $\pm 0.08 \%) 3-N T$ levels decreased significantly.

\section{Quantification of lipid hydroperoxides by tert-butyl hydroperoxide initiated chemiluminescence}

Figure $3 \mathrm{~A}$ shows the levels of lipid hydroperoxides formed in tumor-bearing rats during cachexia progression. The curves of the day 5 , day 10 and day 14 groups were significantly higher $(p<0.0001)$ compared to the control group. Figure $3 \mathrm{~B}$ shows the levels of lipid hydroperoxides in cerebellum of tumor-bearing rats on D5 days treated with inhibitors of CNOS (LNAME) and iNOS (aminoguanidine), respectively day $5 \mathrm{LN}$ and day $5 \mathrm{AG}$. The groups and day $5 \mathrm{LN}$ and day $5 \mathrm{AG}$ curves were significantly lower than group D5.

\section{Discussion}

Tumor growth induces marked changes in the oxidative metabolism of distant tumor-free tissues and organs of the host [24], and these tumor effects on the brain are poorly understood. Cerebellar degeneration is observed in the course of neoplastic diseases (paraneoplastic neurological syndrome), defined as non-metastatic neurological complications [5]. The release of neurotoxic factors by microglia has a triggering role in the development of neurological disorders. After microglia and astrocyte activation, there is a massive release of TNF- $\alpha$, ROS and RNS, especially NO, which contributes to the establishment of neurodegeneration [18]. In low concentrations, NO acts as a powerful lipid peroxidation chain reaction terminator, but its pro-oxidant activity is evidenced when NO is generated in high concentrations [21].

It has been shown that Walker 256 tumor growth causes oxidative stress in the brain [6]. However, the participation of $\mathrm{NO}$ in the cellular damage in the establishment of cerebellar degeneration in the cachectic state has not been demonstrated. Oxidative stress was demonstrated in the hippocampus, cortex and cerebellum of Walker 256 tumor-bearing rats by the determination of thiobarbituric acid reactive substances (TBARS) and enzymatic activities (superoxide dismutase and catalase) [6]. The TBARS technique was used as a marker of oxidative stress evaluation. In this study, a very sensitive tert-butyl hydroperoxide-initiated chemiluminescence assay (CL) was used to analyze the levels of lipid peroxides in the cerebellum of cachectic tumor-bearing rats. This assay indicates that 
the increase in $C L$ is closely related to the oxidative stress previously suffered by the tissue $[10,19]$.

From D5 to D14 there was a progressive body weight loss, compatible to the cachectic syndrome, and it remained even when NOS inhibitors were administered. The same happened for the tumor weight and the progression of the cachexia index, which did not change with the use of inhibitors. The cerebellum weight showed a different behavior. Although there is no statistical difference, day 5 and day 10 showed an increase of weight variation compatible with the establishment of a systemic inflammatory state in cancer disease [20]. This evidence was better observed when $A G$ and $L N$ were administered on day 5 and the cerebellum weight variation decreased.

Although oxidative stress was evidenced, the participation of NO in cachexia-induced cerebellar degeneration remained unclear. In the present study, cachectic state establishment, NO production, 3-NT quantification and lipid peroxidation of the cerebellum were analyzed during progression of the cachectic state in Walker 256 tumor-bearing rats. The cancer-cachectic state of the present model showed that there was significant NO production on D5 in the cerebellum of tumor-bearing rats that decreased when AG and LNAME were used, indicating that both NO production pathways (iNOS and cNOS) are induced. The inhibition by LNAME reduced NO production 1,300 fold compared to AG inhibition. This fact suggests that the NO production through constitutive NOS, mainly nNOS, plays a crucial role in the cerebellum of cachectic rats just before macro alterations start to happen. In chemically controlled NO production by the reaction with potassium iodate [14], NO reacts with $\mathrm{H}_{2} \mathrm{O}_{2}$ in an aqueous phase, giving a stronger oxidizing species, peroxynitrite $\left(\mathrm{ONOO}^{-}\right)$, under basic conditions. CPTIO, a specific NO scavenger [22], was used to provide additional evidence that the object of analysis was in fact NO. The scavenger decreased in a dose-dependent manner the light emission in both biological and chemical systems [23].

Nitrotyrosine (3-NT) is a product of tyrosine oxidation mediated mainly by endogenous peroxynitrite $\left(\mathrm{ONOO}^{-}\right)$. This molecule is a powerful oxidant that interacts with many proteins, which can lead to the formation of nitrotyrosine, the oxidation of thiol groups, lipid peroxidation induction and even DNA damage, as well as the depletion of stored antioxidants [11]. The occurrence of 3-NT in tissues is a marker of $\mathrm{ONOO}^{-}$formation and, therefore, the presence of $\mathrm{NO}$ and $\mathrm{O}_{2}{ }^{\bullet-}$ [12]. Nitrotyrosine was measured in the cerebellum and was coordinated with the increase in NO chemiluminescence on day 5 . Increased levels of 3-NT in the day 10 and day 14 groups can be explained by the capacity of deposits of this oxidized protein on the tissue to induce proteotoxicity. When AG or L-NAME was used in the day 5 group, this protein aggregate decreased, as verified by NO chemiluminescence, which also decreased.

The increase of NO on day 5 is followed by an increase in lipid peroxidation in the cerebellum. The lipid peroxidation evidenced at the beginning of the cachectic state (day 5) may be dependent on the production of NO, and probably its reaction products with oxygen reactive substances [11]. This was confirmed by the use of the inhibitors L-NAME and $A G$, which reduced the NO production and also membrane lipid hydroperoxide formation. Tert-butyl hydroperoxide-initiated $\mathrm{CL}$ was used to evaluate the integral level of nonenzymatic antioxidant defense. A lower level of antioxidant as a consequence of previous oxidative stress corresponds to an accentuated peak and a shift to the left in CL curves, giving a positive correlation between $\mathrm{CL}$ and lipid peroxidation $[15,17]$. In this study, we demonstrated a qualitative difference in all CL curves, indicating alterations in cell membrane properties, such as antioxidant composition, which modifies the kinetics pattern by exhausting the oxidizable species [1].

Michalak et al. [16] demonstrated that circulating TNF- $\alpha$ and MCP-1 (macrophage chemoattractant protein-1), together with decreased levels of insulin and thyroxine, may produce a milieu of factors involved in the mechanism of the development of cerebellar degeneration in cachectic hepatoma-bearing rats, but the overproduction of NO in the cerebellum was not mentioned. The lipid peroxidation profile shows that on all days of tumor progression there was a significant increase in cell membrane lesions. The same pattern of oxidative injury was also observed in muscle of tumor-bearing rats in the same period of cachexia progression [10], and more, that muscular waste is evident, and that oxidative stress is part of the modulation process of ubiquitin-proteasome-induced proteolysis.

According to Keynes et al. [13], the concentration of NO in the cerebellum is dependent on its generation and degradation rate. The consumption of NO by these cells decreases by $50 \%$ when lipid 
peroxidation inhibitors are used. Changes in the activity of the total antioxidants impair the antioxidant defense capacity of the brain and may lead to oxidative stress. Walker 256 tumor growth caused a consistent increase of chemiluminescence in the cerebellum that probably resulted from the changes observed in NO content.

This is the first time that nitric oxide has been related to induced nitrosative and oxidative cerebellar injury in an experimental cancer model that leads to cachexia. It is still unclear whether the role of NO is one of protection or harmful action in cachexia. Nevertheless, this study reveals that in cachexia-induced cerebellar degeneration, nitrosative stress and lipid hydroperoxidation occur in a time-dependent manner, probably induced by the high concentration of NO produced by the cerebellum at the onset of the cachectic state.

\section{Acknowledgments}

Grant support was provided by Coordenação de Aperfeiçoamento de Pessoal de Nível Superior CAPES. We are very grateful to J. A. Vargas for excellent technical assistance.

\section{Disclosure}

\section{Authors report no conflict of interest.}

\section{References}

1. Azorin I, Bella MC, Iborra FJ, Fornas E, Renau-Piqueras J. Effect of tert-butyl hydroperoxide addition on spontaneous chemiluminescence in brain. Free Rad Biol Med 1995; 19: 795-803.

2. Brown GC, Bal-Price A. Inflammatory neurodegeneration mediated by nitric oxide, glutamate, and mitochondria. Mol Neurobiol 2003; 27: 325-355.

3. Calabrese V, Mancuso C, Calvani M, Rizzarelli E, Butterfield DA, Stella AM. Nitric oxide in the central nervous system: neuroprotection versus neurotoxicity. Nat Rev Neurosci 2007; 8: 766-775.

4. Culotta E, Koshland DE Jr. NO news is good-news. Science 1992; 258: 1862-1865.

5. Darnell RB, Posner JB. Paraneoplastic syndromes involving the nervous system. N Engl J Med 2003; 349: 1543-1554.

6. Freitas JJS, Pompéia C, Miyasaka CK, Curi R. Walker-256 tumor growth causes oxidative stress in rat brain. J Neurochem 2001; 77: 655-663.

7. Fukuto, JM, Cho JY, Switzer CH. The chemical properties of nitric oxide and related nitrogen oxides. In: Nitric Oxide - Biology and Pathobiology. Ignarro LJ (ed). Academic Press, San Diego 2000; pp. 23-40.

8. Galla HJ. Nitric oxide, NO, an intercellular messenger. Angew Chem Int Ed Engl 1993; 32: 378-380.
9. Gonzalez-Flecha B, Llesuy S, Boveris A. Hidroperoxide-initiated chemiluminescence: an assay for oxidative stress in biopsies of heart, liver, and muscle. Free Radic Biol Med 1991; 10: 93-100.

10. Guarnier FA, Cecchini AL, Suzukawa AA, Maragno ALGC, Simão ANC, Gomes MD, Cecchini R. Time course of skeletal muscle loss and oxidative stress in rats with Walker 256 solid tumor. Muscle Nerve 2010; 42: 950-958.

11. Halliwell B, Gutteridge JMC. Free Radicals in Biology and Medicine. Oxford University Press, Oxford 2007.

12. Ischiropoulos H, Zhu L, Chen J, Tsai M, Martin JC, Smith CD, Beckman JS. Peroxynitrite-mediated tyrosine nitration catalyzed by superoxide dismutase. Arch Biochem Biophys 1992; 298: 431-437.

13. Keynes RG, Griffiths CH, Hall C, Garthwaite, J. Nitric oxide consumption through lipid peroxidation in brain cell suspensions and homogenates. Biochem J 2005; 387: 685-694.

14. Kikuchi K, Nagano T, Hayakawa H, Hirata Y, Hirobe M. Detection of nitric oxide production from a perfused organ by a luminol- $\mathrm{H}_{2} \mathrm{O}_{2}$ system. Anal Chem 1993; 65: 1794-1799.

15. Llesuy SF, Milei J, Gonzalez-Flecha B, Boveris A. Myocardial damage induced by doxorubicins: hydroperoxide-initiated chemiluminescence and morphology. Free Rad Biol Med 1990; 8: 259-264.

16. Michalak S, Wender M, Michalowska-Wender G. Cachexiainduced cerebellar degeneration: involvement of serum TNF and MCP-1 in the course of experimental neoplastic disease. Acta Neurobiol Exp 2006; 66: 113-122.

17. Oliveira FJA, Cecchini R. Oxidative stress of liver in hamsters infected with Leishmania (L.) chagasi. J Parasitol 2000; 86: 10671072.

18. Pannu R, Singh I. Pharmacological strategies for the regulation of inducible nitric oxide synthase: neurodegenerative versus neuroprotective mechanisms. Neurochem Int 2006; 49: 170-182.

19. Peres PS, Terra VA, Guarnier FA, Cecchini R, Cecchini AL. Photoaging and chronological aging profile: understanding oxidation of the skin. J Photochem Photob B 2001; 103: 93-97.

20. Roxburgh CS, McMillan DC. Role of systemic inflammatory response in predicting survival in patients with primary operable cancer. Future Oncol 2000; 6: 149-163.

21. Rubbo H, Radi R, Trujillo M, Telleri R, Kalyanaraman B, Barnes S, Kirk M, Freeman BA. Nitric oxide regulation of superoxide and peroxynitrite-dependent lipid peroxidation. J Biol Chem 1994; 269: 26066-26075.

22. Terra VA, Souza-Neto F, Pereira RC, Silva TNX, Costa ACC, Luiz RC, Cecchini R, Cecchini AL. Time-dependent reactive species formation and oxidative stress damage in the skin after UVB irradiation. Photochem Photobiol B 2012; 109: 34-41.

23. Terra VA, Souza-Neto FP, Pereira RC, Xavier Da Silva TN, Ramalho LN, Luiz RC, Cecchini R, Cecchini AL. Nitric oxide is responsible for oxidative skin injury and modulation of cell proliferation after 24 hours of UVB exposures. Free Radic Res 2012; 46: 872-882.

24. Ushmorov A, Hack V, Dröge W. Differential reconstitution of mitochondrial respiratory chain activity and plasma redox state by cysteine and ornithine in a model of cancer cachexia. Cancer Res 1999; 59: 3527-3534.

25. Ventrucci G, Mello MAR, Gomes-Marcondes MCC. Proteasome activity is altered in skeletal muscle tissue of tumour-bearing rats fed a leucine-rich diet. Endocr Relat Cancer 2004; 11: 887-895. 